\title{
Análisis espacio-temporal de los cambios previstos en la evapotranspiración de referencia durante el siglo XXI en la Demarcación Hidrográfica del Segura (España) a partir de los modelos climáticos globales MPEH5 y MPEH5C'
}

\author{
Marcos Ruíz-Álvarez², Francisco Gomariz-Castillo³ \\ y Francisco Alonso-Sarría ${ }^{4}$
}

\begin{abstract}
RESUMEN
Se propone una metodología para realizar proyecciones espacio-temporales de la evapotranspiración de referencia $\left(\mathrm{ET}_{0}\right)$ y el posible impacto de cambio climático en cuencas semiáridas. El estudio se desarrolla en la Demarcación Hidrográfica del Segura, utilizando para estimar la $\mathrm{ET}_{0}$ el modelo de Hargreaves calibrado mensualmente mediante el método de Allen. Se parte de una regionalización de temperaturas máximas y mínimas a partir de los modelos globales MPEH5 y MPEH5C y los escenarios de emisión SRESA2, SRESA1B, SRESB1 y E1. Se ha evaluado la tendencia temporal y la distribución espacial de $\mathrm{ET}_{0}$ mediante Theil-Sen, Mann-Kendall y 3 métodos de interpolación (regresión lineal múltiple, krigreado ordinario y regresión-krigeado). Se observa un incremento significativo en los 4 escenarios entre los 2,73 mm para el SRESA2 y los 0,63 mm para E1. Se observa además un patrón espacial caracterizado por un aumento mayor de la $\mathrm{ET}_{0}$ en zonas de cabecera y menor en la zona litoral.
\end{abstract}

Palabras clave: Cambio climático, Evapotranspiración de referencia, Demarcación Hidrográfica del Segura, MPEH5.

\begin{abstract}
A methodology is proposed to carry out spatio-temporal projections of reference evapotranspiration $\left(\mathrm{ET}_{0}\right)$, and to analyse the possible impact of climate change, on semi-arid basins. The study is carried out in the Segura Hydrographic Demarcation, using the Hargreaves model, calibrated monthly following the Allen method, to estimate $\mathrm{ET}_{0}$. The input information is a regionalization of maximum and minimum temperatures based on the global models MPEH5 and MPEH5C and the emission scenarios SRESA2, SRESA1B, SRESB1 and E1. The temporal trend and spatial distribution of $\mathrm{ET}_{0}$ variation were evaluated using the Theil-Sen estimator and Mann Kendall test, and 3 methods of interpolation (multiple linear regression, ordinary kriging and regression-kriging). A significant increase is observed in the 4 scenarios that oscillate between $2.73 \mathrm{~mm}$ in SRESA2 and $0.63 \mathrm{~mm}$ in E1. There is also a spatial pattern that shows a greater increase in $\mathrm{ET}_{0}$ in headwaters and a smaller increase in the coastal zone.
\end{abstract}

Keywords: Climate Change, Reference Evapotranspiration, Segura Hydrographic Demarcation, MPEH5.

\footnotetext{
Este artículo ha sido desarrollado en el marco del Proyecto de Investigación "Actividades de Apoyo Científico al Proceso de Planificación Hidrológica y Cooperación Europea", financiado por la Confederación Hidrográfica del Segura, MAPAMA (Gobierno de España). Este trabajo se ha hecho en parte con financiación del proyecto "El papel de los mercados del agua en la gestión integrada de los recursos hídricos en las cuencas deficitarias" (Ref. 19325/PI/2015) concedido en el marco de la convocatoria "Ayudas a la realización de proyectos de investigación destinadas a grupos competitivos" de la Fundación Séneca, Agencia de Ciencia y Tecnología de la Región de Murcia. Artículo recibido el 10 de abril de 2017, aceptado el 12 de octubre de 2017 y corregido el 8 de noviembre de 2017. 
El cambio climático de origen antrópico ha sido objeto de numerosos estudios, como el del Grupo Intergubernamental de Expertos sobre el Cambio Climático (Intergovernamental Panel on Climate Change, IPCC), y ha generado cierta alarma social. A pesar de las incertidumbres, es indudable que en las últimas décadas se han producido importantes anomalías y cambios en el clima sin precedentes en los últimos siglos. El quinto informe del IPCC (2013) (AR5) identifica diversos cambios en numerosas variables del sistema climático a partir de observaciones en superficie o desde satélite y de simulaciones mediante modelos climáticos y atribuye, con una probabilidad superior al 95\%, más de la mitad del aumento observado en la temperatura media global en superficie durante el periodo 1951-2010 a factores antrópicos. Así, por ejemplo, los datos de temperatura de la superficie terrestre y oceánica, combinados y promediados globalmente, muestran un calentamiento de $0,85^{\circ} \mathrm{C}$ durante el período 1880-2012 (IPCC, 2013:3). En el AR5 se destaca además que cada uno de los tres últimos decenios ha sido sucesivamente más cálido que cualquier otra década anterior desde 1850.

La Agencia Estatal de Meteorología del Gobierno de España (AEMET) ha realizado proyecciones regionalizadas para el siglo XXI de precipitación y de temperatura máxima para las Demarcaciones Hidrográficas españolas (AEMET, 2015). Dicho trabajo parte de los resultados de los modelos globales de la quinta fase del Proyecto de Comparación de Modelos Acoplados (CMIP5), utilizados en la elaboración del AR5. Estos resultados son reinterpolados mediante 2 técnicas de regionalización estadística (análogos y regresión lineal). Los resultados muestran una gran incertidumbre asociada al modelo global utilizado y en menor medida a la técnica de regionalización.

El Cuadro Nº 1 recoge la variación media en la Demarcación Hidrográfica del Segura (DHS) de los modelos globales del CMIP5 para tres escenarios de emisión definidos en el AR5 (RCP 4.5, RCP 6.0 y RCP 8.5). Estos escenarios se caracterizan por el cálculo aproximado que hacen del forzamiento radiativo total en el año 2100 en relación con 1750, oscilando éste entre los $4,5 \mathrm{~W} / \mathrm{m}^{2}$ para RCP 4.5 y los $8,5 \mathrm{~W} / \mathrm{m}^{2}$ para RCP 8.5. Los escenarios RCP 4.5 y RCP 6.0 son de estabilización y el RCP 8.5, es un escenario con un nivel muy elevado de emisiones. Para el RCP 4.5 el forzamiento se estabiliza en 2100, mientras que para RCP6.0 y RCP8.5 sigue aumentando más allá de esa fecha (IPCC, 2013: 27).

\section{Cuadro No 1}

Variación media de la precipitación y temperatura prevista para el periodo 2041-2070 en la demarcación hidrográfica del Segura durante el periodo de referencia 1961-1990

\begin{tabular}{|c|c|c|c|c|}
\hline \multirow{2}{*}{ Escenario } & \multicolumn{2}{|c|}{ Precipitación (\%) } & \multicolumn{2}{|c|}{ Temperatura $\left({ }^{\circ} \mathrm{C}\right)$} \\
\hline & Análogos & Regresión & Análogos & Regresión \\
\hline RCP 8.5 & -12 & -15 & 2,7 & 3 \\
\hline RCP 6.0 & -9 & -13 & 1,9 & 2,1 \\
\hline RCP 4.5 & -7 & -11 & 1,9 & 2,1 \\
\hline
\end{tabular}

Fuente: Elaboración propia a partir de los datos disponibles en el portal web de la AEMET.

Los datos regionalizados mediante regresión son ligeramente superiores, en todos los casos, a los obtenidos a partir del método de análogos. El máximo descenso de las precipitaciones, así 
como el máximo ascenso de las temperaturas, está previsto para el escenario RCP8.5. El descenso de las precipitaciones disminuye paulatinamente conforme lo hace el forzamiento radiativo estimado por los escenarios. Sin embargo, en el caso de las temperaturas, los escenarios RCP 4.5 y RCP 6.0 prevén la misma variación para el periodo 2041-2070.

La evapotranspiración es uno de los procesos más importantes dentro del ciclo del agua. Existen diferentes definiciones de dicho proceso entre la que se encuentra la evapotranspiración de referencia $\left(\mathrm{ET}_{0}\right.$ ) que se define como "la tasa de evapotranspiración de una superficie de referencia, que ocurre sin restricciones de agua. La superficie de referencia es un cultivo hipotético de pasto, con una altura asumida de $0,12 \mathrm{~m}$, con una resistencia superficial fija de $70 \mathrm{~s} \mathrm{~m}^{-1} \mathrm{y}$ un albedo de 0,23" (Allen et al., 2006). El conocimiento de su evolución futura, junto a la de las precipitaciones, es el punto de partida para poder realizar un estudio de los efectos del cambio climático en la gestión de los sistemas de recursos hídricos. Este tipo de estudios son fundamentales para evaluar cambios potenciales en los recursos hídricos en territorios como las zonas semiáridas del sureste español, con precipitaciones irregulares y escasas. La Demarcación Hidrográfica del Segura (DHS), área de estudio del presente trabajo, es un buen ejemplo de este tipo de ambientes, con un elevado déficit hídrico de $400 \mathrm{Hm}^{3} /$ año (CHS, 2015) y una fuerte presión antrópica derivada de usos como la agricultura de regadío o el turismo.

El importante incremento de las temperaturas previsto para la DHS en AEMET (2015), aproximadamente $3{ }^{\circ} \mathrm{C}$ para el escenario RCP 8.5 en el periodo 2041-2070 con respecto al periodo 1961-1990, provocaría un importante aumento de la ET. Este hecho, junto al posible descenso de las precipitaciones, podría acentuar el déficit hídrico actual. Conocer la magnitud espacio-temporal del aumento de la $\mathrm{ET}_{0}$ durante el siglo XXI es uno de los puntos de partida para cuantificar la reducción de las aportaciones en régimen natural y evaluar los efectos del cambio climático en la gestión de los recursos hídricos.

Son diversos los estudios que han analizado la evolución espacio-temporal de la $\mathrm{ET}_{0}$ desde la década de 1960 hasta la actualidad (Li et al., 2012; Vicente-Serrano et al., 2014; Vicente-Serrano et al., 2016; Gao et al., 2017). Pero el efecto del cambio climático sobre la ET 0 ha sido menos estudiado que en el caso de temperatura o precipitación.

Sin embargo, en los últimos cinco años se han desarrollado algunos estudios acerca de la variación espacio-temporal prevista para la $\mathrm{ET}_{0}$ durante el siglo XXI en diversas áreas del planeta. Li et al. (2012) analizaron esta variación en la Meseta de Loess (China) durante el siglo XXI y regionalizaron, aplicando el método Statistical Downscaling Method (SDSM), los resultados obtenidos por el modelo global HadCM3 en 48 estaciones utilizando los escenarios SRESA2 y SRESB2. Estos autores detectaron tendencias positivas significativas en 47 de las 48 estaciones analizadas para el escenario SRESA2 durante el periodo 2071-2100; para el escenario SRESB2 dicha tendencia significativa se detectó en 32 estaciones. Tao et al. (2015) obtuvieron para la cuenca del río Xiangjiang (China) proyecciones futuras de la $\mathrm{ET}_{0}$ utilizando SDSM, aunque en este caso las regionalizaciones se realizaron a partir de resultados obtenidos por los modelos pertenecientes al CMIP5 y los escenarios RCP4.5 y RCP8.5. En dicho estudio se detectó una tendencia positiva significativa en la media anual de la $\mathrm{ET}_{0}$ para los 2 escenarios analizados en los periodos 2041-2070 y 2071-2100. Tanto Li et al. (2012) como Tao et al. (2015) interpolaron los valores de la $\mathrm{ET}_{0}$ con el método de ponderación inversa a la distancia (IDW). Terink et al. (2013) analizaron la 
evolución espacio-temporal prevista de la $\mathrm{ET}_{0}$ en el Medio Oriente y el Norte de África, obteniendo proyecciones hasta el año 2050 mediante el método de Hargreaves. Como información de partida se utilizaron regionalizaciones de temperatura máxima y mínima referidas al escenario SRESA1B, obtenidas mediante SDSM a partir de los resultados de los 9 modelos globales del proyecto CMIP3 con mejores resultados en la región (Shongwe et al., 2009, 2011). La evaluación de los modelos se basó en la media de los coeficientes de correlación mensuales y el error cuadrático medio entre los datos procedentes del Climatic Research Unit (CRU) y los simulados por los modelos globales.

En España, el informe técnico del CEDEX (2011) analiza los cambios previstos para el siglo XXI en la precipitación, la $\mathrm{ET}_{0}$, y la escorrentía total para tres periodos temporales distribuidos a lo largo del siglo XXI, 2011-2040, 2041-2070 y 2071-2100, tomando como referencia el periodo 1961-1990. Se emplearon 6 proyecciones regionalizadas, obtenidas a partir de distintos modelos globales y técnicas de regionalización. Los resultados se refieren a 2 escenarios de emisión (SRESA2 y SRESB2). La ET, se estimó utilizando la ecuación de Hargreaves por necesitar solo valores de temperatura máxima y mínima diarias, disponibles normalmente en las series de escenarios de cambio climático; a pesar de ello es uno de los métodos que mejor aproximan la ET real (Di Stefano \& Ferro, 1997; López-Urrea et al., 2006).

Sin embargo, la ecuación de Hargreaves subestima la $\mathrm{ET}_{0}$ en regiones secas o elevadas y la sobreestima en zonas bajas (Droogers \& Allen, 2002). Entre las variables cuya no inclusión en el modelo de Hargreaves podría reducir su exactitud se han destacado: radiación (Hargreaves, 1994), ratio entre la presión atmosférica y la presión a nivel del mar (Allen, 1995), precipitación

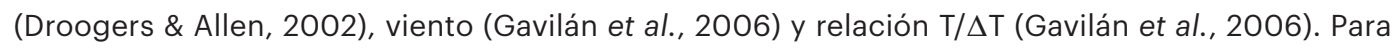
solventar el problema, suele calibrarse para aproximarla a las estimaciones obtenidas con la ecuación de Penman-Monteith FAO, considerada el modelo más adecuado para estimar $\mathrm{ET}_{0}$ (Allen et al., 1994; Subburayan et al., 2011) pero que requiere un gran número de variables meteorológicas, no disponibles en la mayoría de los observatorios meteorológicos ni en las series de las proyecciones de cambio climático.

Las estimaciones puntuales de la $\mathrm{ET}_{0}$ pueden ser útiles para la gestión local del regadío, pero no son representativas de áreas amplias en regiones heterogéneas (Kidron \& Zohar, 2010). Algunos estudios han tratado de interpolar valores de $\mathrm{ET}_{0}$ utilizando variables auxiliares como la elevación (Mardikis et al., 2005; Vicente-Serrano et al., 2007), o sin tenerlas en cuenta (Häntzschel et al., 2005). Li et al. (2012) o Terink et al. (2013) obtienen la distribución espacial de los cambios de $\mathrm{ET}_{0}$; sin embargo, CEDEX (2011) estima solo valores agregados.

Los objetivos de este estudio son: Analizar el impacto espacial y temporal del cambio climático sobre la $\mathrm{ET}_{0}$ en la DHS; para ello se han obtenido proyecciones de $\mathrm{ET}_{0}$ para el siglo XXI y 4 escenarios de emisión: SRESA2, SRESA1B, SRESB1 y E1. Para estimar la ET, se ha utilizado el método de Hargreaves (Hargreaves \& Sanami, 1985), calibrado siguiendo la metodología propuesta en Gomariz-Castillo (2016). En segundo lugar, interpolar los valores de ET, utilizando variables auxiliares de fácil obtención que puedan actuar como proxies de las variables meteorológicas relevantes, antes mencionadas, y que no se incluyen en el modelo de Hargreaves. 


\section{Materiales y métodos}

La DHS se localiza en el sureste de la Península Ibérica. Cuenta con una superficie de 19.025 $\mathrm{km}^{2}$, e incluye territorios situados en cuatro comunidades autónomas: prácticamente la totalidad de la Región de Murcia y parcialmente territorios de la Comunidad Valenciana, Andalucía y Castilla-La Mancha (Figura No 1). La cuenca principal de la Demarcación, con una superficie de 15.177 $\mathrm{km}^{2}$, es la del río Segura, con una longitud de $325 \mathrm{~km}$. Además, se incluyen las cuencas vertientes al Mar Mediterráneo $\left(2.733 \mathrm{~km}^{2}\right)$ al sur y este y las cuencas endorreicas de Yecla y Corralrrubio al Norte $\left(1.115 \mathrm{~km}^{2}\right)$.

Figura $\mathrm{N}^{\circ} 1$

Ámbito territorial de la DHS y distribución espacial de las estaciones termométricas utilizadas

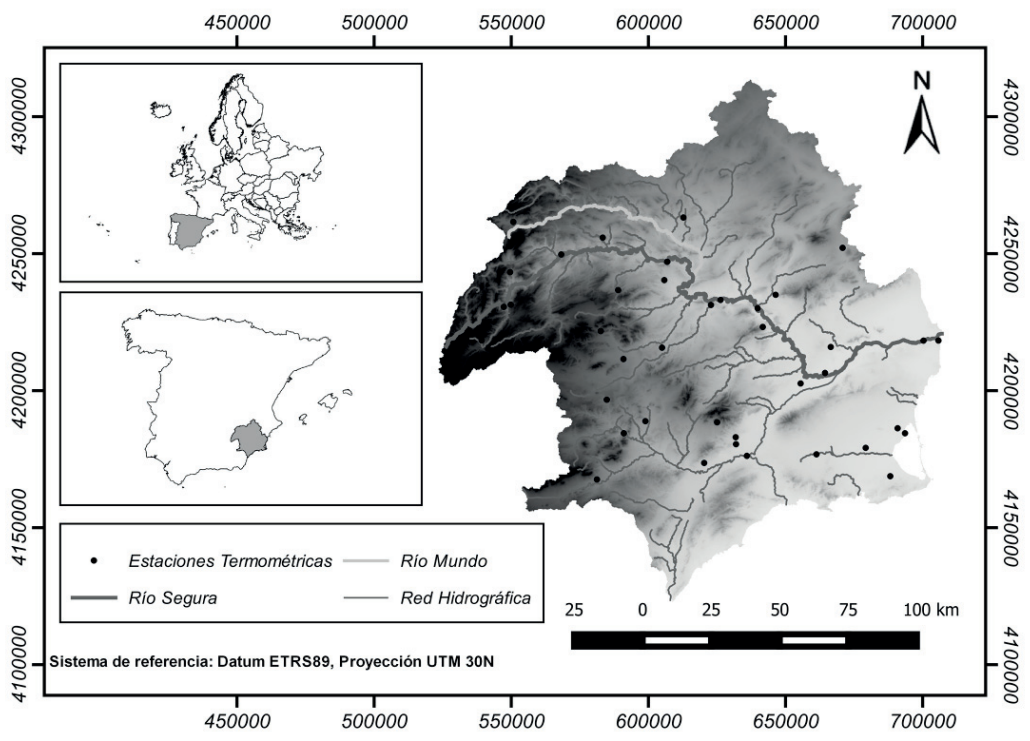

Fuente: Elaboración propia.

Geológicamente, corresponde a las Cordilleras Béticas que siguen una dirección ENE-OSO. Sin embargo los sistemas montañosos en la Cuenca del Segura, especialmente al Noroeste, tienden a formar un arco por un cambio de dirección, primero a NE-SO y después, rápidamente, a NO-SE. La causa de este arco, denominado Arco Cazorla-Alcaraz-Hellín, es un sistema de fallas dextrosas (Rodríguez-Pascua \& De Vicente, 2001). Este arco es también el responsable de la forma curvada de los sistemas de drenaje (Figura $\mathrm{N}^{\circ} 1$ ). Las mayores altitudes aparecen en el sector noroeste: Revolcadores $(2.017 \mathrm{~m})$ o Sierra de las Cabras $(2.083 \mathrm{~m})$, aunque algunas de las sierras situadas al sur alcanzan elevaciones importantes a escasa distancia de la costa: sierras de La Carrasquilla (879 m de altitud a $11 \mathrm{~km}$ de la costa), Carrascoy (1.085 m a $39 \mathrm{~km}$ de la costa), Sierra de La Torrecilla (974 m a 38 km de la costa) o Sierra Espuña (1.584 m a 40 km de la costa). La litología de estas elevaciones es predominantemente carbonatada en el norte y centro de la cuenca y 
metamórfica en el Sur. Entre ellas aparecen depresiones que han sido rellenadas por sedimentos generalmente de tipo margoso (Romero Díaz \& López Bermúdez, 2009).

En relación con el clima resulta especialmente relevante la posición latitudinal de la DHS en la transición entre los climas templado-húmedos, asociados a las borrascas del frente polar, y los secos, asociados al anticiclón de las Azores, así como su localización en el margen occidental del continente europeo (López Bermúdez \& Alonso Sarría, 2001). Estos factores implican un clima mediterráneo con veranos cálidos e inviernos suaves y elevada insolación, entre 2800 y $3000 \mathrm{~h} /$ año (López Bermúdez \& Alonso Sarría, 2001). La orografía influye sobre la distribución espacial de las temperaturas, que aumentan desde las sierras del Noroeste $\left(10^{\circ} \mathrm{C}\right)$ hasta el litoral $\left(18^{\circ} \mathrm{C}\right)$. El régimen anual de temperaturas presenta un mínimo invernal en los meses de diciembre y enero, mientras que los máximos corresponden a los meses de julio y agosto (CHS, 2015:130). La cercanía al mar suaviza las temperaturas estivales e invernales, especialmente cerca de la costa. Se observa un aumento de la continentalidad desde la franja costera hasta la zona septentrional, tanto por la distancia al mar como por la presencia de sucesivas alineaciones montañosas (Conesa García \& Alonso Sarría, 2006).

El anticiclón de las Azores determina la mayor parte de las situaciones atmosféricas (Balairón, 1997) y en consecuencia la baja frecuencia de situaciones atmosféricas favorables a las precipitaciones, la precipitación media anual en el periodo 1980/81-2011/12 fue de 375 mm (CHS, 2015:129). Su distribución espacial está muy ligada al relieve, con valores ligeramente superiores a los 1.000 $\mathrm{mm} / a n ̃ o$ en las sierras situadas al noroeste, que disminuyen drásticamente en dirección $\mathrm{N}$-S hasta valores próximos a los $200 \mathrm{~mm} / \mathrm{año}$ en el litoral suroccidental.

Aunque el clima mediterráneo suele definirse por un máximo de precipitación en invierno, el área de estudio lo presenta en otoño. La gran cantidad de territorio continental que deben atravesar las masas de aire húmedo atlántico antes de llegar a la DHS, y la existencia del arco montañoso antes mencionado, limitan enormemente el número de borrascas atlánticas que alcanzan la cuenca en invierno y la precipitación que éstas producen. Los relieves de dicho arco son el único sector de la misma en que se producen los máximos de precipitación invernales característicos de los climas mediterráneos (Conesa García \& Alonso Sarría, 2006).

Por otro lado, a finales de verano y principios de otoño, la elevadas temperaturas del mar mediterráneo (Millán et al., 1995; Quereda Sala et al., 2006) junto a la presencia de aire frío y presión baja en altura como depresiones aisladas en niveles altos (DANA) o como una vaguadas en la circulación zonal, pueden generar episodios de ciclogénesis que, debido a la circulación antihoraria, acaba entrando en la Península y alcanzando los relieves costeros, que presentan elevadas altitudes cerca de la costa. Además, su disposición alargada y en forma de arco hace que las masas de aire que entran con dirección E-O alcancen fácilmente los relieves del interior, forzando un ascenso suficiente para generar inestabilidad (Llasat, 2009). Esta inestabilidad provoca un mecanismo de realimentación con la entrada de más aire mediterráneo cargado de humedad, provocando precipitaciones de alta intensidad que pueden producir avenidas e inundaciones (López Bermúdez et al., 2002).

La distribución de la evapotranspiración potencial $\left(\mathrm{ET}_{\mathrm{p}}\right)$ es similar a la del resto de variables climáticas. La Confederación Hidrográfica del Segura estima a partir del modelo de Thornthwaite un valor medio de la $\mathrm{ET}_{\mathrm{p}}$ anual en la DHS de $993 \mathrm{~mm}$, muy superior a los $341 \mathrm{~mm}$ de evapotrans- 
piración real media (CHS, 2015:92); los valores más bajos aparecen en la zona de cabecera (zona noroeste de la Demarcación), con los mayores relieves y las temperaturas más bajas, y tienden a aumentar hacia la costa, siguiendo la línea NO-SE que marca el relieve, con máximos en las sierras litorales, en la Vega Baja y la desembocadura del río Segura.

El resultado conjunto es una región climática con elevada aridez en la que las fluctuaciones y la irregularidad son la norma (López Bermúdez \& Alonso Sarría, 2001). Bajo estas circunstancias, la vegetación natural se adapta adoptando una estructura en mosaico y mediante mecanismos de resilencia (González Barberá et al., 1997). Tradicionalmente la agricultura se concentraba en las vegas del río Segura y sus principales afluentes. Éstos se disponen formando una serie de arcos entre relieves generalmente calizos, que permiten la aparición de acuíferos de cierta importancia que dieron lugar a un gran número de manantiales (López Bermúdez et al., 2014) que alimentaron pequeñas huertas tradicionales. La combinación de factores favorables para la agricultura (insolación y temperatura) ha permitido a lo largo del siglo XX el desarrollo de una agricultura muy productiva en comparación con el resto de España (Colino Sueiras et al., 2014) que supone un porcentaje del PIB mayor que en el resto de España (Calvo García-Tornel, 2006). Sin embargo, debido a la aridez de la cuenca, resulta muy dependiente del regadío, por lo que se ha necesitado un elevado grado de regulación (Calvo García-Tornel, 2006), el trasvase de agua de zonas vecinas (Morales Gil et al., 2005), y la sobreexplotación de acuíferos (García Aróstegui et al., 2014). El 46\% de los manantiales registrados en 1916 han desaparecido por la sobreexplotación de los acuíferos y la disminución de la precipitación en forma de nieve, debido al aumento térmico ligado al cambio climático (López Bermúdez et al., 2014).

La agricultura y el turismo son dos de sus motores económicos, por lo que los recursos hídricos son fundamentales para su desarrollo. El previsible incremento futuro de las temperaturas, junto al posible descenso de las precipitaciones, podrían ocasionar importantes impactos ambientales como el descenso de la biodiversidad vegetal y animal o la disminución de los recursos hídricos tanto superficiales como subterráneos (MMA, 2005; CEDEX, 2012).

\section{Fuentes de información y proceso de trabajo}

Para obtener las proyecciones de $\mathrm{ET}_{0}$ en el siglo XXI se han utilizado los datos de temperaturas máximas y mínimas derivados de las proyecciones regionalizadas de cambio climático en España de la AEMET (Morata Gasca, 2014). De la información disponible en este proyecto, tan solo cinco modelos (MPEH5-MPEH5C, EGMAM-EGMAM2, y IPCM4-IPCM4V2, INGV y BCCRBCM2) contienen información para todo el siglo XXI y los cuatro escenarios de emisión considerados (SRESA2, SRESA1B, SRESB1 y E1). Tras una evaluación previa del ajuste de las series respeto al periodo de referencia (Cuadro $\mathrm{N}^{\circ}$ 2) se han seleccionado los datos de MPEH5-MPEH5C, con un mejor ajuste y menor error y sesgo.

El modelo MPEH5 (Roeckner, 2007) forma parte del proyecto ENSEMBLES-Stream1 (ES1) y el modelo MPEH5C (Roeckner, 2008) del proyecto ENSEMBLES-Stream2 (ES2). Las proyecciones realizadas a partir del modelo MPEH5 utilizan los escenarios de emisión SRESA2, SRESB1 y SRESA1B (Nakicenovic et al., 2000), que representan diferentes cambios demográficos, sociales, económicos, tecnológicos y medioambientales. Las proyecciones realizadas a partir del modelo MPEH5C utilizan el escenario E1 para el periodo 2001-2099. E1 es un escenario de mitigación agresivo 


\section{Cuadro $\mathrm{N}^{\circ} 2$}

Ajuste medio entre las proyecciones regionalizadas y los datos observados en las estaciones termométricas utilizadas. Periodo de referencia 1961-1990

\begin{tabular}{|l|r|r|r|r|r|r|}
\hline \multirow{2}{*}{ Modelo } & \multicolumn{2}{|c|}{ R2 } & \multicolumn{2}{c|}{ RMSE } & \multicolumn{2}{c|}{ \%Sesgo } \\
\cline { 2 - 7 } & $\mathrm{T}^{\circ}$ mín. & \multicolumn{1}{c|}{$\mathrm{T}^{\circ}$ máx. } & $\mathrm{T}^{\circ}$ mín. & \multicolumn{1}{c|}{$\mathrm{T}^{\circ}$ máx. } & $\mathrm{T}^{\circ}$ mín. & $\mathrm{T}^{\circ}$ máx. \\
\hline MPEH5 & 0.93 & 0.92 & 1.45 & 1.9 & 2.8 & 1.3 \\
\hline EGAM & 0.81 & 0.82 & 2.8 & 3.25 & -9.2 & -4.5 \\
\hline IPCM4 & 0.91 & 0.87 & 2.29 & 2.95 & -15.4 & -7.8 \\
\hline INGV & 0.92 & 0.92 & 1.75 & 2.19 & 4.4 & 4.1 \\
\hline BCCRBCM2 & 0.92 & 0.91 & 2.25 & 2.74 & -14.1 & -8.3 \\
\hline
\end{tabular}

Fuente: Elaboración propia a partir de las series regionalizadas de la AEMTE y los datos la red de observatorios de la AEMET.

definido en el proyecto ENSEMBLES (van der Linden \& Mitchell, 2009), cuyo objetivo es evitar que se superen $2^{\circ} \mathrm{C}$ de calentamiento global medio respecto a los niveles pre-industriales.

La regionalización de la AEMET se basa en el método de análogos (Petisco de Lara, 2008) utilizando 374 estaciones termométricas. Se han utilizado los datos de las 39 estaciones ubicadas en la DHS (Figura $N^{\circ}$ 1) en los periodos 1971-2000 (utilizado como serie de referencia) y 2001-2099 y un intervalo temporal diario. Se detectaron incoherencias en el 0,03"\% de los datos y se depuraron rellenando los datos ausentes y anómalos con el método de Guijarro (2014), que compara cada serie original con series de referencia obtenidas utilizando una correlación basada en distancias ponderadas.

El proceso de trabajo se desarrolla bajo software Open Source, generando un procedimiento estandarizado y reproducible con el que pueden almacenarse, gestionar y procesar grandes volúmenes de información. La información de los escenarios climáticos se ha almacenado y procesado utilizando PostgreSQL; para el almacenamiento, procesamiento y análisis de la información espacial se ha utilizado el SIG GRASS y el programa R. La programación y automatización de las tareas se ha realizado bajo los lenguajes de Bash y $R$.

\section{Obtención de proyecciones de $E T_{0}$ para el siglo XXI}

La $\mathrm{ET}_{0}$ se estimó mediante el método de Hargreaves (Hargreaves y Samani, 1985):

$$
E T_{0, H G}=C R_{a}\left(T_{\max }-T_{\min }\right)^{0,5}(\mathrm{Tmed}+17,78)
$$

Donde $E T_{0, H G}$ es la $E T_{0}$ calculada mediante la ecuación de Hargreaves ( $m m$ dia $^{-1}$ ); $C=0,0023$ es un coeficiente empírico; $R_{a}$ es la radiación extraterrestre en unidades de evaporación equivalente (mm día-1), calculada de acuerdo a Samani (2000); $T_{\text {med }} T_{\max }$ y $T_{\min }$ son la temperatura media, máxima y mínima diaria $\left({ }^{\circ} \mathrm{C}\right)$. Una vez obtenidos los datos diarios de la $\mathrm{ET}_{0}$ mediante el método de Hargreaves, se han agregado a escala mensual, seleccionada como escala temporal de trabajo. 
En este trabajo se ha utilizado el método propuesto por Gomariz-Castillo (2016) para estimar los valores de la $\mathrm{ET}_{0}$ calculados con el modelo de Penman-Monteith FAO a partir de los de Hargreaves mediante 12 modelos de regresión lineal mensuales en los que $b_{0}=0$. Por tanto, la ecuación de calibración para cada mes quedaría como:

$$
E T_{0, P M}=b_{1} \cdot E T_{0, H G}
$$

Este método mejora significativamente el ajuste de Hargreaves a la serie de referencia y muestra cuando el modelo de Hargreaves sobreestima $\left(b_{1}<1\right)$ o infraestima $\left(b_{1}>1\right)$ los valores de ETo obtenidos por el método de Penman-Monteith-FAO.

\section{Impacto del cambio climático sobre la $E T_{0}$}

Para analizar el impacto del cambio climático sobre la $\mathrm{ET}_{0}$ en la DHS, se ha estudiado la evolución anual durante el siglo XXI de los cambios en dicha variable con respecto al periodo de referencia 1971-2000. Los datos de la $\mathrm{ET}_{0}$ se han obtenido a partir del promedio de los valores anuales de dicha variable en las 39 series disponibles. Finalmente, se han comparado, a escala mensual, estacional y anual, los valores de la $\mathrm{ET}_{0}$ estimados para cada uno de los escenarios en el periodo 2041-2070 con los valores de la ET, para el periodo 1971-2000.

La tendencia temporal anual de las series se ha estimado a partir de una regresión robusta de Theil-Sen y el contraste Mann-Kendall para evaluar su significación. Estos dos métodos no paramétricos, descritos en Cannarozzo et al. (2006), se han utilizados para analizar tendencias temporales por su mayor robustez ante el incumplimiento de los supuestos necesarios en los métodos paramétricos tradicionales. Las diferencias en los valores medios anuales y estacionales de los escenarios se han evaluado mediante un ANOVA de dos vías de un modelo lineal mixto, considerando como factor fijo los escenarios y como factor aleatorio la variable año. Para las comparaciones múltiples entre los diferentes escenarios se ha utilizado el test de Tukey para la diferencia de las diferencias, basado en la distribución del rango estudentizado.

\section{Distribución espacial de $E T_{0}$}

Las estimaciones de la $\mathrm{ET}_{0}$ obtenidas en los 39 observatorios se han interpolado para los periodos 1971-2000 y 2041-2070 utilizando 3 métodos diferentes: regresión lineal múltiple (MRLM) estimando los parámetros mediante mínimos cuadrados generalizados, krigeado ordinario (OK) y regresión-krigeado (RK); la implementación del proceso utilizado puede consultarse en Gomariz-Castillo y Alonso-Sarría (2013).

MRLM es un método de interpolación global que define una relación funcional entre la variable dependiente $\left(\mathrm{ET}_{0}\right)$ y un conjunto de variables ambientales distribuidas espacialmente. Para evaluar la normalidad de los residuos se ha utilizado la prueba de Kolmogorov-Smirnov y para evaluar la homocedasticidad la prueba de Breush-Pagan. Se han utilizado 7 variables ambientales como predictores: 1) elevación sobre el nivel del mar (m), obtenida del Modelo Digital de Elevaciones (MDE) a escala 1:25.000 del Instituto Geográfico Nacional de España con una resolución de 25 $m, 2)$ distancia $(m)$ a la costa y masas de agua, 3) irradiación potencial mensual (Wh. $\left.\mathrm{m}^{-2} \cdot \mathrm{mes}^{-1}\right)$, obtenida con la metodología propuesta por Hofierka \& Súri (2002) y 4) la transformación inversa y logarítmica de la elevación y de la distancia a la costa. 
Estas variables pueden actuar como proxies de algunas de las variables meteorológicas relevantes en el cálculo de la $\mathrm{ET}_{0}$ pero no incluidas en la ecuación de Hargreaves. Así la irradiación potencial es un proxy de la radiación global; la elevación lo es de la ratio entre la presión atmosférica y la presión atmosférica al nivel del mar o de la magnitud del viento; finalmente, la distancia a la costa actúa como proxy de la relación $T / \Delta T$, la humedad, o incluso también de la magnitud del viento o la nubosidad. En el primer caso, la amplitud térmica está relacionada con la distancia al mar, el efecto suavizador que esta tiene sobre la temperatura contribuye al aumento de las mínimas y al descenso de las máximas y, por tanto, a un descenso de la amplitud térmica. En cuanto a la humedad, la cercanía al mar implica la entrada de aire húmedo marino en las zonas de costa, efecto que va reduciéndose hacia el interior. En áreas costeras, el viento sopla con más intensidad cuando llega del mar debido a la ausencia de obstáculos en éste; en el interior la presencia de obstáculos reduce su magnitud con la excepción de las áreas de montaña, pero ese efecto ya es tenido en cuenta al incluir la elevación como predictor. Finalmente, la entrada de aire húmedo favorece la nubosidad en las áreas costeras, efecto que disminuye hacia el interior. Sin embargo, los fenómenos convectivos pueden generar, en la estación cálida, un aumento de la nubosidad en el interior. La posible falta de linealidad de algunas de estas relaciones se compensa por la introducción como predictores de las transformaciones inversa y logarítmica de la elevación y la distancia al mar. Aunque algunas de estas relaciones pueden ser discutibles, en un trabajo reciente Gomariz Castillo et al. (2017) constataron, en el mismo área de estudio, que la distancia a la costa es un factor fundamental para explicar los errores cometidos por el método de Hargreaves al estimar $\mathrm{ET}_{0} \mathrm{y}$, por tanto, a la hora de calibrarlo para aproximar los valores obtenidos por el modelo PM-FAO.

El análisis y selección de variables predictoras se ha llevado a cabo en dos pasos: 1) Se ha evaluado la multicolinealidad entre variables predictoras a partir del factor de inflación de la varianza (VIF), eliminando de forma iterativa las variables con, umbral propuesto por O'brien (2007); 2) regresiones por pasos minimizando el criterio de información de Akaike.

OK (Matheron, 1971) es un método de interpolación local geoestadístico basado en medias ponderadas, donde los coeficientes de ponderación se estiman a partir de la función semivariograma. En este trabajo, el semivariograma experimiental se ajusta de forma automática al modelo teórico de Mattern mediante el algoritmo propuesto por Hiemstra et al. (2008).

RK (Odeh et al., 1994; Hengl et al., 2004) es un método mixto que permite integrar las dos aproximaciones anteriores mediante la interpolación espacial, utilizando OK, de los errores del modelo de regresión.

Para evaluar y comparar el error cometido por los modelos, se ha utilizado una validación cruzada uno a uno (LOOCV). El error cometido en promedio se ha estimado mediante la Raíz del Error Cuadrático Medio (RMSE).

Finalmente, los mapas de variación de la $\mathrm{ET}_{0}$ con respecto al periodo de referencia 1971-2000 se han obtenido a escala mensual, utilizando los modelos de interpolación para los que se minimiza el RMSE de la validación cruzada. Para ello se han restado los valores de $\mathrm{ET}_{0}$ mensuales del periodo histórico a los obtenidos para el periodo 2041-2070. Finalmente, los mapas de variación mensual de la $\mathrm{ET}_{0}$ se han agregado a escala estacional y anual. Estos últimos se presentan en la Figura No 5 como resumen de los resultados obtenidos. 


\section{Evolución anual de los cambios durante el siglo XXI en la ET, en la DHS}

Los escenarios analizados prevén un aumento de la $\mathrm{ET}_{0}$ estadísticamente significativo ( $\mathrm{p}$-valor $<0,001$ en el contraste de Mann-Kendall) en la DHS durante el siglo XXI (Figura No 2). Este aumento es especialmente importante para los escenarios SRESA2 y SRESA1B $(2,72$ y 2,26 mm/año respectivamente) que asumen a partir de 2050 mayor concentración de gases de efecto invernadero. Por el contrario, en el periodo 1971-2000 no se observa un aumento, siendo la media de las 39 series de 1.243 mm/año. Para SRESA2 el aumento alcanza los 100 mm (8\%) en 2050 y supera ligeramente los $200 \mathrm{~mm}(16 \%)$ al final del siglo XXI. El escenario SRESA1B prevé un incremento similar durante el siglo XXI, aunque con una estabilización al final del periodo en torno a los 175 $\mathrm{mm}(14 \%)$.

Figura $\mathrm{N}^{\circ} 2$

Evolución de la $\mathrm{ET}_{0}$ observada en la serie histórica y en los 4 escenarios de cambio climático considerados. El modelo de tendencia lineal se ha obtenido mediante el estimador Theil-Sen

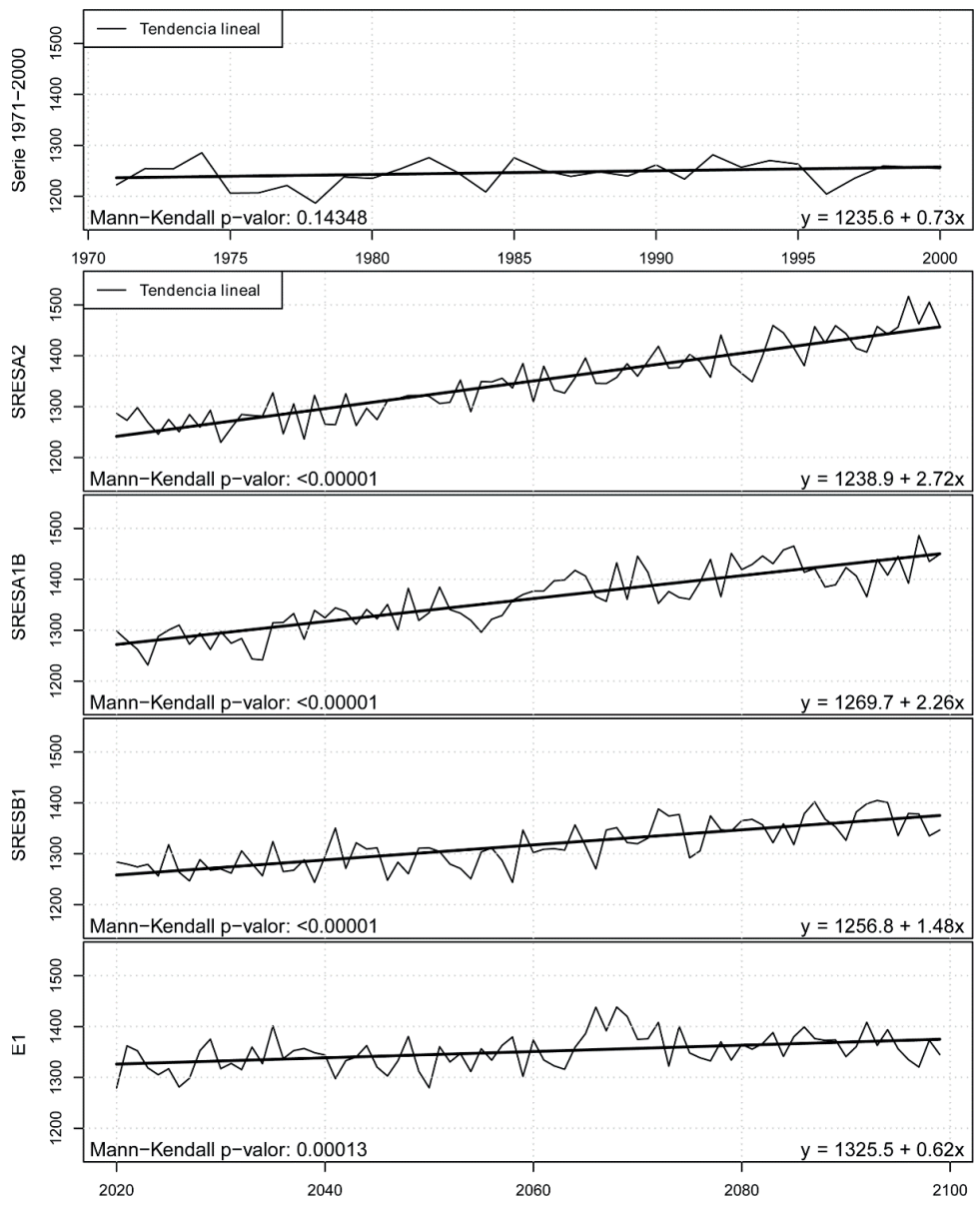

Fuente: Elaboración propia. 
El escenario SRESB1 prevé un aumento de la $\mathrm{ET}_{0}(1,48 \mathrm{~mm} / \mathrm{año})$, aunque menor que en los anteriores, que se estabilizaría a finales del periodo. En 2050 el aumento de la $\mathrm{ET}_{0}$ se situaría alrededor de los $50 \mathrm{~mm}$ (4\%), mientras que a finales de siglo estaría en torno a $125 \mathrm{~mm}$ (10\%). Para E1 (escenario de mitigación) se observa un menor incremento $(0,62 \mathrm{~mm} / \mathrm{año})$. El aumento previsto de la $\mathrm{ET}_{0}$ hasta 2050 para $\mathrm{E} 1$ es superior al resto de los escenarios, alcanzando los $150 \mathrm{~mm}(12 \%)$ en la década de 2070, desde donde desciende hasta estabilizarse al final del periodo en valores similares a SRESB1. Este comportamiento se debe a que en E1, donde se definen las mayores emisiones en 2010 (van der Linden \& Mitchell, 2009:16), los valores de temperatura pronosticados son mayores al resto de escenarios desde el inicio de la serie (con un valor en el intercepto de $\mathrm{ET}_{0}=1.325,5 \mathrm{~mm} / \mathrm{año}$ ) hasta 2030, tal y como puede verse en Morata Gasca (2014:145); otro factor que puede afectar en las diferencias entre E1 y el resto en las primeras décadas del siglo XXI éste se obtiene a partir del modelo MPEH5C y el resto a partir de MPEH5.

\section{Variación mensual, estacional y anual de $\mathrm{ET}_{0}$ en la DHS en el periodo 2041-2070 con respecto al periodo de referencia 1971-2000}

El Cuadro $N^{\circ} 3$ muestra los valores medios de la variación de la $\mathrm{ET}_{0}$ en el periodo 2041-2070 a escala mensual, estacional y anual. Los principales aumentos se concentran entre mayo y septiembre, especialmente en los meses estivales. Entre noviembre y febrero el ascenso medio no alcanza en ningún caso los $5 \mathrm{~mm}$.

\section{Cuadro $\mathrm{N}^{\circ} 3$}

Variación media de la $\mathrm{ET}_{0}$ a escala mensual, estacional y anual en la DHS para el periodo 2041-2070. Variación en mm. Periodo de referencia 1971-2000

\begin{tabular}{|l|r|r|r|r|l|r|r|r|r|}
\hline \multirow{2}{*}{ Mes } & \multicolumn{4}{|c|}{ Escenario } & \multirow{2}{*}{ Mes } & \multicolumn{4}{|c|}{ Escenario } \\
\cline { 2 - 6 } & SRESA2 & SRESA1B & SRESB1 & E1 & & SRESA2 & SRESA1B & SRESB1 & E1 \\
\hline ENE & 3,8 & 3,4 & 2,3 & 1,4 & OCT & 7,1 & 8,9 & 4,8 & 7,7 \\
\hline FEB & 2,8 & 3,7 & 3 & 2,6 & NOV & 3,2 & 3,8 & 1,6 & 2,2 \\
\hline MAR & 4,4 & 7,6 & 3,1 & 7,1 & DIC & 3,4 & 3,1 & 2,2 & 0,6 \\
\hline ABR & 5,1 & 9,9 & 1,1 & 10,7 & Estación & SRESA2 & SRESA1B & SRESB1 & E1 \\
\hline MAY & 11,8 & 13 & 6,6 & 16,4 & PRI & 21,3 & 30,5 & 10,8 & 34,1 \\
\hline JUN & 11,4 & 14,5 & 7,6 & 15,1 & VER & 35,7 & 46,9 & 26,8 & 44,4 \\
\hline JUL & 11,8 & 16,4 & 9,6 & 14,2 & OTO & 18,7 & 24,1 & 12,7 & 20,8 \\
\hline AGO & 12,5 & 16 & 9,5 & 15,1 & INV & 10 & 10,2 & 7,5 & 4,7 \\
\hline SEP & 8,4 & 11,4 & 6,3 & 10,8 & Anual & 85,7 & 111,7 & 57,8 & 104 \\
\hline
\end{tabular}

Fuente: Elaboración propia.

A escala anual, los escenarios SRESA1B y E1 son los más pesimistas en cuanto a la evolución de la $\mathrm{ET}_{0}$, ya que estiman un ascenso medio anual ligeramente superior a los $100 \mathrm{~mm}$ (9\%). Las 
proyecciones para SRESB1 son las más optimistas, el ascenso medio anual no supera los $60 \mathrm{~mm}$ (5\%). El verano es la estación en que se alcanza el mayor ascenso medio, que oscila entre $47 \mathrm{~mm}$ para el escenario SRESA1B y $27 \mathrm{~mm}$ para el SRESB1. En primavera el ascenso medio previsto de la $\mathrm{ET}_{0^{\prime}}$ varía entre los $34 \mathrm{~mm}$ del escenario E1 y los $11 \mathrm{~mm}$ del escenario SRESB1. En otoño, el incremento medio de la $\mathrm{ET}_{0}$ oscila entre los $24 \mathrm{~mm}$ del escenario SRESA1B y los $13 \mathrm{~mm}$ estimados para el escenario SRESB1. En invierno, el ascenso medio de la $\mathrm{ET}_{0}$ se sitúa en torno a los $10 \mathrm{~mm}$ para los escenarios SRESA1B y SRESA2, mientras los otros dos no superan esta cifra.

La Figura No 3 muestra las medias anuales y los intervalos de confianza al 95\%, así como las medias estacionales de $\mathrm{ET}_{0}$; la agrupación que se presenta (grupos a a d) resume los contrastes a posteriori de Tukey. La asignación de la serie histórica al grupo a indica que el valor medio de $\mathrm{ET}_{0}$ es significativamente menor que para los 4 cuatro escenarios. Entre los escenarios analizados, la asignación de SRESB1 (correspondiente a un escenario con emisiones bajas) al grupo b indica que el valor medio de $\mathrm{ET}_{0}=1303 \pm 12 \mathrm{~mm} / \mathrm{año}$ es significativamente menor al resto de escenarios. El escenario con un valor medio de $E T_{0}$ más elevado es SRESA1B $\left(E T_{0}=1358 \pm 14 \mathrm{~mm} / \mathrm{año}\right.$ ), significativamente mayor al resto de escenarios, exceptuando a E1. SRESA1B, caracterizado por unas

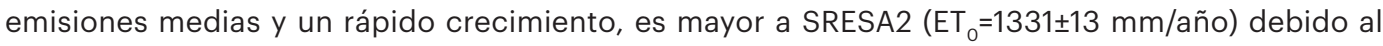
periodo de tiempo en el que se comparan, siendo superado por éste último a partir de 2080. Finalmente, en el caso de E1, el valor medio anual tan elevado $\left(E_{0}=1349 \pm 15 \mathrm{~mm} / \mathrm{año}\right)$ puede deberse a los factores comentados en el apartado anterior.

En lo referente al ciclo estacional, aunque el patrón es similar a la serie histórica para los cuatro escenarios, los aumentos de la $\mathrm{ET}_{0}$ son consistentemente mayores en verano que en invierno. En todos los casos se observan diferencias significativas entre estaciones, con valores máximos en verano y mínimos en invierno.

Figura $\mathrm{N}^{\circ} 3$

Medias anuales de la $\mathrm{ET}_{0}$ e intervalos de confianza al 95\%. Las letras sobre los resultados anuales muestran los grupos de escenarios no significativamente distintos, ordenadas de menor a mayor valor promedio de la $\mathrm{ET}_{0}$ (a-d)
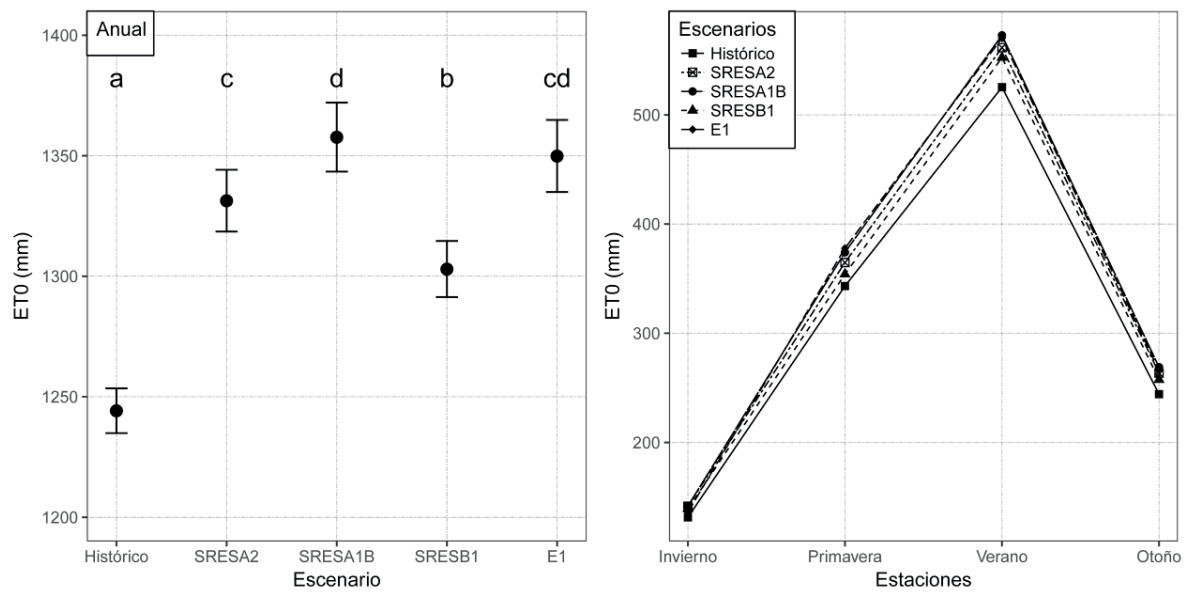

Fuente: Elaboración propia. 
La Figura $\mathrm{N}^{\circ} 4$ muestra las medias estacionales, intervalos de confianza al 95\% y grupos homogéneos de los contrastes a posteriori de Tukey de la serie histórica y de los cuatro escenarios analizados. Los contrastes de ANOVA son significativos entre escenarios en las cuatro estaciones ( $p$-valor $<<0,00001$ en todos los casos). Las pautas anuales se reproducen por lo general en todas las estaciones salvo en invierno, donde $\mathrm{E} 1$ muestra un aumento de $\mathrm{ET}_{0}$ inferior al resto de los escenarios, tal y como puede verse en el Cuadro $N^{\circ} 3$.

En todas las estaciones la serie histórica es significativamente menor a los cuatro escenarios y SREA1B es el escenario con valores medios más elevados excepto en primavera, donde E1 tiene el valor medio más elevado. Al igual que ocurría a nivel anual, SRESB1 suele ser el escenario con un menor aumento de $\mathrm{ET}_{0}$ respecto al escenario histórico, siendo significativamente menor en primavera, verano y otoño (grupo b); en cambio, en invierno no se observan diferencias significativas entre los cuatro escenarios.

Figura $\mathrm{N}^{\circ} 4$

Medias estacionales de $\mathrm{ET}_{0}$ e intervalos de confianza al 95\%. Las letras sobre los resultados anuales muestran los grupos de escenarios no significativamente distintos
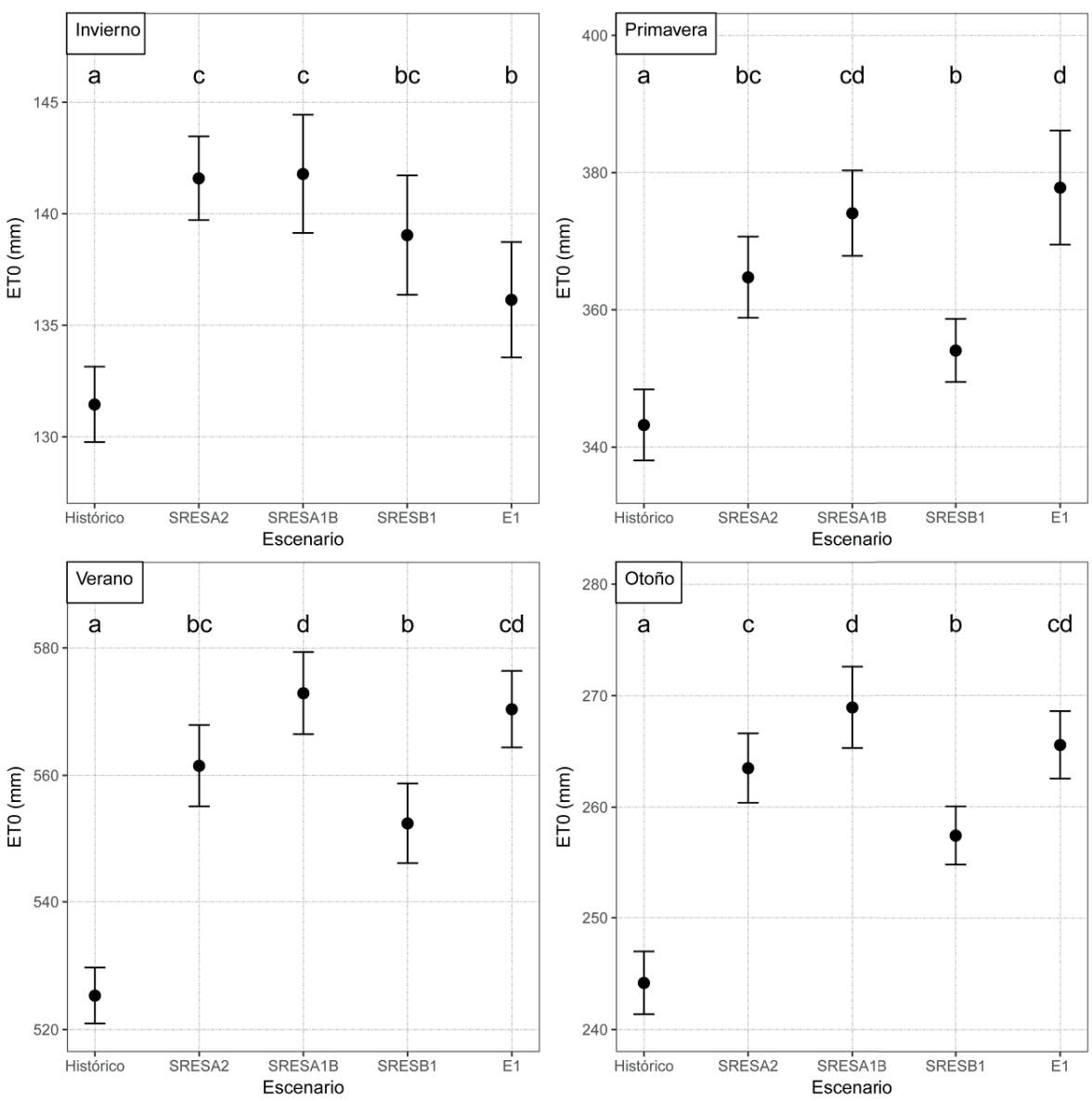

Fuente: Elaboración propia. 
De los resultados obtenidos se infieren repercusiones considerables en la disponibilidad hídrica. Si bien la evolución de la precipitación está sujeta a una mayor incertidumbre que en el caso de las temperaturas, con trabajos en los que incluso parece aumentar la precipitación anual en la zona de trabajo en la primera del siglo XXI (CEDEX, 2012:7), en AEMET (2014:145) se constata para la Región de Murcia un descenso en todos los escenarios, a excepción de E1 (con una pendiente en la tendencia próxima a 0 ).

El estudio realizado por el CEDEX (2011:135) obtiene para el periodo 2041-2070 un aumento de la $\mathrm{ET}_{0}$ anual del $11 \%$ para el escenario SRESA2 y del 10\% para el escenario SREA1B. Estas cifras son ligeramente superiores a las obtenidas en el presente trabajo. Las diferencias observadas pueden deberse al uso en este estudio de una colección actualizada de datos regionalizados de la AEMET o a diferencias en la obtención de los parámetros de calibración. En Gomariz-Castillo (2016) se utiliza un mayor número de estaciones para calibrar Hargreaves y una metodología para su interpolación basada en modelos RK, permitiendo incluir efectos locales en las interpolaciones no abordados en la escala de trabajo del CEDEX (2011).

\section{Distribución espacial de $\mathrm{ET}_{0}$}

El cuadro $\mathrm{N}^{\circ} 4$ muestra los modelos de interpolación que minimizan el RMSE cometido en validación cruzada en cada uno de los meses. Por tanto, éstos son los modelos escogidos para generar los mapas mensuales de $\mathrm{ET}_{0}$. En el $45 \%$ de los casos se ha seleccionado OK y en el $38 \%$ MRLM. La selección del tipo de interpolación parece depender más del mes de que se trate que del período o escenario analizado, indicando un patrón en la distribución espacial de la ET 0 caracterizado por una mayor influencia de los factores ambientales considerados en aquellos meses en los que se utiliza MRLM, y un mayor peso de la autocorrelación espacial en los casos en los que selecciona OK. Para los meses de febrero, marzo, agosto y octubre, todos los mapas se han obtenido utilizando el modelo MRLM. Por su parte, mediante OK se han obtenido todos los mapas de enero y diciembre (meses con una menor $\mathrm{ET}_{0}$ ) y septiembre (relacionado probablemente por una mayor influencia de otros factores, como la nubosidad o la humedad ambiental, factores derivados de las mayores precipitaciones en este mes). Algo parecido sucede en abril, mes donde suele producirse un máximo secundario en la precipitación; en este mes se ha seleccionado OK o RK en todos los casos. El modelo RK, es el que menos se ha empleado en este trabajo $(17 \%$ de los mapas realizados). Con este modelo se han obtenido 4 mapas en el mes de noviembre, 3 en el mes de mayo y 1 para los meses de abril, junio y julio.

La distancia a la costa y la elevación son las variables más frecuentemente incorporadas en los modelos (en el $79 \%$ y el $67 \%$ de ellos respectivamente); la transformación inversa de la elevación y la logarítmica de la distancia aparecen en un $58 \%$ de los modelos; la transformación inversa de la distancia a la costa, la radiación mensual y la transformación logarítmica de la elevación han sido las variables menos utilizadas en los modelos (52\%, $45 \%$ y 33 "\% respectivamente). Los coeficientes de las variables predictoras obtenidos, muestran un patrón espacial de la $\mathrm{ET}_{0}$ caracterizado por una tendencia a disminuir conforme aumenta la altitud y la distancia a la costa.

La Figura No 5 resume la distribución espacial de la variación de la $\mathrm{ET}_{0}$ a partir de su agregación anual. El mayor aumento aparece en todos los casos en la cabecera de los ríos Segura y Mundo y desciende en dirección noroeste-sureste hasta la zona litoral. 
Cuadro $\mathrm{N}^{\circ} 4$

Resumen de los modelos utilizados para la generación de los mapas de la $\mathrm{ET}_{0}$

\begin{tabular}{|l|c|c|c|c|c|c|c|c|c|c|c|c|c|}
\hline \multirow{2}{*}{ Periodo } & \multirow{2}{*}{ Escenario } & \multicolumn{10}{|c|}{ Modelo que minimiza RMSE } \\
\cline { 3 - 13 } & & ENE & FEB & MAR & ABR & MAY & JUN & JUL & AGO & SEP & OCT & NOV & DIC \\
\hline 1971-2000 & Histórico & OK & MRLM & MRLM & OK & MRLM & OK & OK & MRLM & OK & MRLM & MRLM & OK \\
\hline \multirow{5}{*}{$2041-2070$} & SRESA2 & OK & MRLM & MRLM & OK & RK & OK & OK & MRLM & OK & MRLM & RK & OK \\
\cline { 2 - 13 } & SRESA1B & OK & MRLM & MRLM & OK & RK & OK & RK & MRLM & OK & MRLM & RK & OK \\
\cline { 2 - 13 } & SRESB1 & OK & MRLM & MRLM & RK & RK & OK & OK & MRLM & OK & MRLM & RK & OK \\
\cline { 2 - 12 } & E1 & OK & MRLM & MRLM & OK & MRLM & RK & OK & MRLM & OK & MRLM & RK & OK \\
\hline
\end{tabular}

Fuente: Elaboración propia.

Figura $\mathrm{N}^{\circ} 5$

Distribución espacial de la variación anual de la $\mathrm{ET}_{0}$ en la DHS prevista para los cuatro escenarios analizados en el periodo 2041-2070. Periodo de referencia 1971-2000

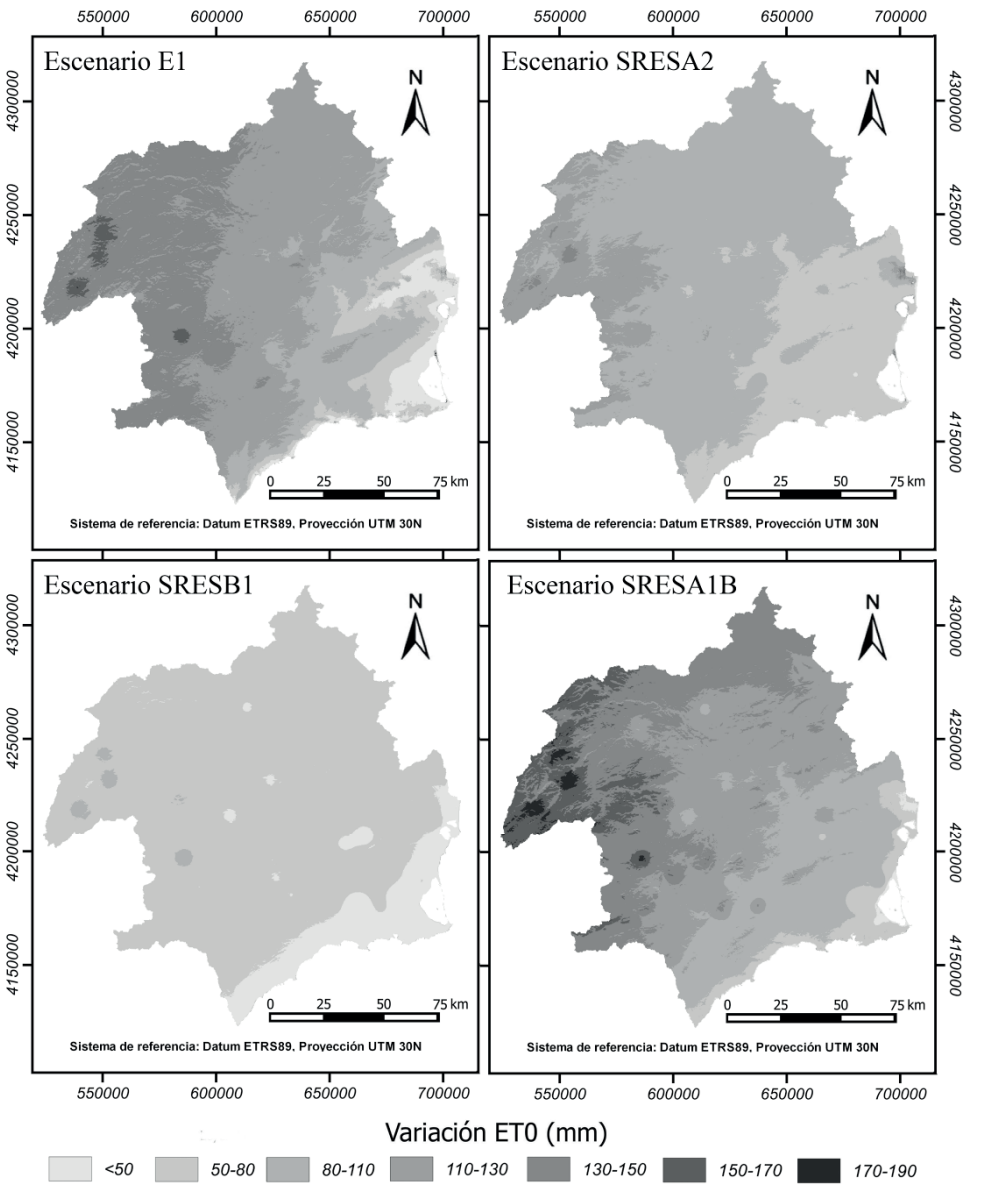

Fuente: Elaboración propia. 
Figura $\mathrm{N}^{\circ} 6$

Distribución espacial de la variación estacional en primavera y otoño de la ETO en la DHS prevista para los escenarios sresb1 y sresa1b en el periodo 2041-2070. Periodo de referencia 1971-2000

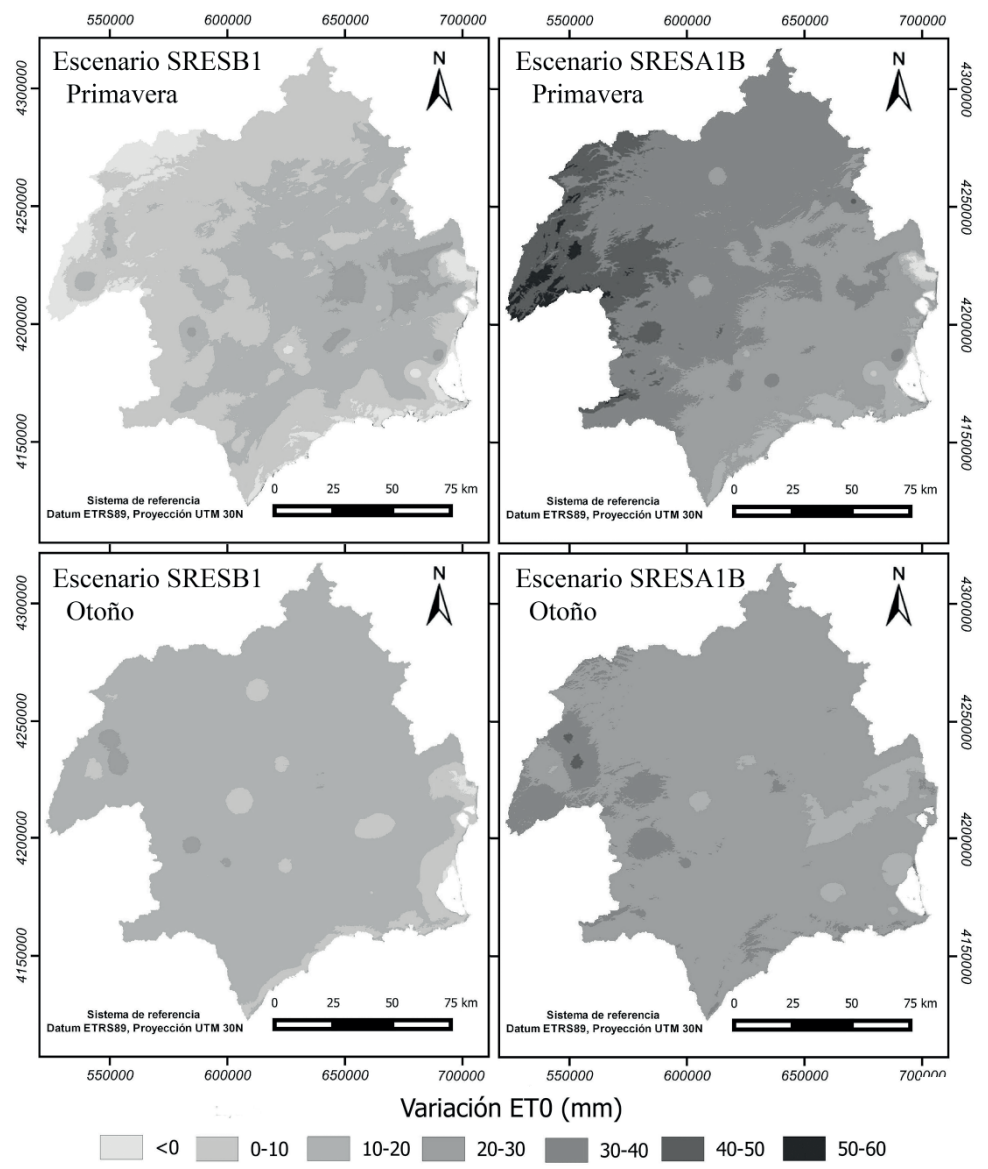

Fuente: Elaboración propia.

El escenario SRESA1B, donde el ascenso de la $\mathrm{ET}_{0}$ supera los $100 \mathrm{~mm}$ en gran parte de la DHS, es el más pesimista. Los mayores ascensos se alcanzan en la cabecera, donde se superan los 150 $\mathrm{mm}$. Este aumento disminuye en dirección noroeste-sureste, hacia el litoral, donde el ascenso de la $\mathrm{ET}_{0}$ se sitúa en torno a los $60 \mathrm{~mm}$. El escenario E1 prevé una evolución similar, aunque en este caso en zonas litorales y prelitorales de la Demarcación el ascenso se sitúa ligeramente por debajo de los $50 \mathrm{~mm}$ y el área donde el aumento supera los $150 \mathrm{~mm}$ es más reducida. El escenario SRESA2 es más optimista que los analizados anteriormente, estimando un ascenso máximo de la $\mathrm{ET}_{0}$ en torno a los $130 \mathrm{~mm}$ en zonas de cabecera, y superándose los $100 \mathrm{~mm}$ en una extensa área de la mitad occidental de la DHS y en su extremo septentrional. En zonas costeras se observa un aumento en torno a los $50 \mathrm{~mm}$. Por último, para el escenario SRESB1, el más optimista, se prevé un ascenso de la $\mathrm{ET}_{0}$ máximo en torno a los $90 \mathrm{~mm}$ en el extremo occidental de la Demarcación, oscilando el aumento entre 50 y $60 \mathrm{~mm}$ en la mayor parte del área de estudio, a excepción de las zonas litorales, donde se registra un aumento inferior a este rango. 
El patrón espacial intra-anual observado en promedio coincide con el apartado anterior, siendo los escenarios SRESA1B el que obtiene mayores incrementos en todas las estaciones a excepción de primavera, donde es superado por E1. Debido a la extensión de este trabajo, en la Figura No 6 se incluye tan solo la distribución estacional en primavera y otoño (estaciones con mayor pluviosidad de la zona de estudio) y los escenarios SRESB1 y SRESA1B.

El mayor aumento de la $\mathrm{ET}_{0}$ a escala estacional se observa en verano y el menor en invierno. En los escenarios SRESA1B y E1 el incremento de $\mathrm{ET}_{0}$ supera los $70 \mathrm{~mm}$ en algunos puntos de cabecera. En el escenario SRESB1, el más optimista, el ascenso supera ligeramente los $40 \mathrm{~mm}$ en algunos puntos de la cabecera. En primavera el ascenso apenas supera los $50 \mathrm{~mm}$ en SRESA1B en zonas de cabecera. El escenario SRESB1 es el más optimista al estimar ascensos que tan solo superan los $20 \mathrm{~mm}$ en algunos puntos de la Vega Media del Segura. En otoño, el mayor ascenso se prevé en el escenario SRESA1B, superando los $30 \mathrm{~mm}$ en zonas de cabecera y en zonas prelitorales. En SRESB1 el ascenso de la $\mathrm{ET}_{0}$ apenas llega a $20 \mathrm{~mm}$ en zonas de cabecera. En invierno se registran los valores más bajos de $\mathrm{ET}_{0}$.

El patrón espacial observado puede agravar todavía más la situación deficitaria de los recursos hídricos. Los mayores aportes proceden de la zona de cabecera de la cuenca del Segura, coincidente con las zonas en las que más aumentará la $\mathrm{ET}_{0}$. El cambio detectado puede afectar además a aspectos como el régimen en los caudales ecológicos, de vital importancia en la zona de cabecera para la preservación del entorno menos alterado en la DHS, o la disminución de la recarga subterránea en esta zona, principalmente calcárea.

\section{Conclusiones}

Las proyecciones realizadas con todos los escenarios muestran un aumento significativo de la $\mathrm{ET}_{0}$ durante el siglo XXI. Sin embargo, el aumento detectado ha sido algo inferior al encontrado en CEDEX (2012), aunque las repercusiones en la zona de estudio pueden ser elevadas.

En este estudio se detecta un incremento elevado de la $\mathrm{ET}_{0^{\prime}}$, sobre todo en los escenarios SRESA2 y SRESA1B caracterizados por emisiones elevadas o medias, con más de $2 \mathrm{~mm} /$ año, frente a un incremento de 1,45 mm/año en el escenario SRESB1 (de emisiones bajas) o de tan solo 0,63 $\mathrm{mm} / \mathrm{año}$ en el caso del escenario de mitigación E1, inferior al incremento de la serie histórica.

Esta diferencia es evidente al comparar el periodo histórico 1971-2000 con los cuatro escenarios; en todos los casos, la serie histórica es significativamente inferior al resto, siendo el escenario SRESB1 significativamente inferior al resto de escenarios de emisión; en este caso, SRESA1B es en el que se observa un mayor valor medio anual, por encima de SRESA2, aunque a partir de 2080 el valor medio en éste último es superior.

El mismo patrón se observa a escala intra-anual. Aunque el mayor incremento se produce en verano, menos relevante por la escasez de las precipitaciones, el incremento detectado en otras estaciones es bastante pronunciado, aumentando significativamente la evapotranspiración real.

En lo referente a la distribución espacial los mayores aumentos de $\mathrm{ET}_{0}$ se registran en las cabeceras de los ríos Segura y Mundo, disminuyendo en dirección noroeste-sureste hasta el litoral. Las 
principales aportaciones en régimen natural de la DHS proceden de este sector, donde además se encuentran los principales embalses de abastecimiento de la Demarcación.

En la mayoría de los mapas de $\mathrm{ET}_{0^{\prime}}$ los modelos más utilizados han sido OK y MRLM. El uso de OK frente a MRLM o RK puede deberse a la menor influencia de las variables ambientales utilizadas frente a otros factores no considerados en los meses en los que se selecciona (principalmente enero y diciembre) En el caso de MRLM y RK, la distancia a la costa y la elevación fueron las variables predictoras más utilizadas en los modelos, con una tendencia de $\mathrm{ET}_{0}$ a disminuir conforme aumenta la elevación y la distancia a la costa. Entre las ventajas del uso de modelos basados en MRLM destaca la posibilidad de evaluar los efectos no funcionales de las variables ambientales y la generación de modelos de distribución con capacidad de considerar efectos locales de los predictores.

Por último, nos gustaría destacar las ventajas de implementar la metodología bajo programas Open Source. Permite la reproducibilidad a otras zonas en las que hemos iniciado este tipo de estudios, la mejora del proceso mediante la comparación de modelos de regresión no lineal, o la inclusión de nueva información como variables ambientales climáticas y series basadas en otros modelos regionales. Las únicas limitaciones en este sentido son la disponibilidad de datos meteorológicos y los recursos informáticos.

\section{Referencias bibliográficas}

AGENCIA ESTATAL DE METEOROLOGÍA (AEMET). Servicios climáticos. Proyecciones climáticas para el siglo XXI, 2015. Disponible en Internet:

http://www.aemet.es/es/serviciosclimaticos/cambio_climat

ALLEN, R. Evaluation of procedures for estimating mean monthly solar radiation from air temperature, technical report. Roma: United Nations Food and Agricultural Organization (FAO), 1995.

ALLEN, R.G.; SMITH, M.; PERRIE, A. \& PEREIRA, L.S. An update for the calculation of reference evapotranspiration. ICID Bulletin, 1994, N 43, p. 1-34.

ALLEN, R.G; PEREIRA, L.S; RAES. D. y SMITH, M. Evapotranspiración del cultivo. Guías para la determinación de los requerimientos de agua de los cultivos. Estudio FAO Riego y Drenaje 56, Roma: United Nations Food and Agricultural Organization, 2006.

BALAIRÓN, L. El clima mediterráneo y sus características en el contexto de la circulación general atmosférica. En: IBAÑEZ, B.L.; VALERO GARCÉS, B.L. y MACHADO, C. (coordinadores). El paisaje mediterráneo a través del espacio y el tiempo. Implicaciones en la desertificación. Logroño: Geoforma Ediciones, 1997, p. 131-160.

CALVO GARCÍA-TORNEL, F. Sureste Español: regadío, tecnologías hidráulicas y cambios territoriales. Scripta Nova. Revista Electrónica de Geografía y Ciencias Sociales, 2006, Vol. X, No 218. Disponible en Internet: http://www.ub.edu/geocrit/sn/sn-218-04.htm

CANNAROZZO, M.; NOTO, L.V. \& VIOLA, F. Spatial distribution of rainfall trends in Sicily (19212000). Physics and Chemistry of the Earth, 2006, Vol. 31, N 18, p. 1201-1211. 
CENTRO DE ESTUDIOS Y EXPERIMENTACIÓN DE OBRAS PÚBLICAS (CEDEX). Evaluación del impacto del cambio climático en los recursos hídricos en régimen natural, informe técnico. Madrid: Ministerio de Agricultura, Alimentación y Medio Ambiente, 2011.

CENTRO DE ESTUDIOS Y EXPERIMENTACIÓN DE OBRAS PÚBLICAS (CEDEX). Estudio de los impactos del cambio climático en los recursos hídricos y en las masas de agua, informe técnico. Madrid: Ministerio de Agricultura, Alimentación y Medio Ambiente, 2012.

COLINO SUEIRAS, J.; MARTÍNEZ-CARRASCO PLEITE, F. y MARTÍNEZ PAZ, J.M. El impacto de la PAC renovada sobre el sector agrario de la Región de Murcia. Murcia: Consejo Económico y Social de la Región de Murcia, 2014.

CONESA GARCÍA, C. y ALONSO SARRÍA, F. El clima de la Región de Murcia. En: CONESA GARCíA, C. (editor). El medio físico de la Región de Murcia. Murcia: Editum, 2006, p. 95-128.

CONFEDERACIÓN HIDROGRÁFICA DEL SEGURA (CHS). Plan Hidrológico de la Cuenca del Segura 2015-2021. Madrid: Ministerio de Agricultura y Pesca, Alimentación y Medio Ambiente, 2015.

DI STEFANO, C. \& FERRO, V. Estimation of Evapotranspiration by Hargreaves Formula and Remotely Sensed Data in Semi-arid Mediterranean Areas. Journal of Irrigation and Drainage Engineering, $1997, N^{\circ} 68$, p. 189-199.

DROOGERS, P. \& ALLEN, R.G. Estimating reference evapotranspiration under inaccurate data conditions. Irrigation and Drainage Systems, 2002, Vol. 16, N 1, p. 33-45.

GAO, Z; HE, J.; DONG, K \& LI, X. Trends in reference evapotranspiration and their causative factors in the West Liao River basin, China. Agricultural and Forest Meteorology, 2017, № 232, p.106-117.

GARCÍA ARÓSTEGI, J.L.; SENENT ALONSO, M.; MARTÍNEZ VICENTE, D. y ARAGÓN RUEDA, R. La sobreexplotación de acuíferos. En: SENENT ALONSO, M. y GARCÍA ARÓSTEGUI, J.L. (editores). Sobreexplotación de acuíferos en la cuenca del Segura. Evaluación y perspectivas. Murcia: Instituto Euromediterráneo del Agua, 2014, p. 63-132.

GAVILÁN, P.; LORITE, I.; TORNERO, S. \& BERENGENA, J. Regional calibration of Hargreaves equation for estimating reference ET in a semiarid environment. Agricultural Water Management, 2006, Vol. 81, N 3, p. 257-281.

GUIJARRO, J.A. User's Guide to Climatol Versión 2.2. Baleares: AEMET, 2014.

GOMARIZ-CASTILLO, F. Estimación de variables y parámetros hidrológicos y análisis de su influencia sobre la modelización hidrológica: Aplicación a los modelos de Témez y Swat. Murcia: Universidad de Murcia, Tesis Doctoral, 2016.

GOMARIZ-CASTILLO, F. \& ALONSO-SARRÍA, F. An R script to model monthly climatic variables with MRLM to be used in hydrological modeling in River Segura basin. In: The R User Conference, Albacete, 2013. 
GOMARIZ-CASTILLO, F.; ALONSO-SARRÍA, F. \& CABEZAS-CALVO-RUBIO, F. Calibration and spatial modelling of daily ETO in semiarid areas using Hargreaves equation. Earth Science Informics, 2018; $N^{\circ} 3$.

GONZÁLEZ BARBERÁ, F.; LÓPEZ BERMÚDEZ, F. y ROMERO DÍAZ, A. Cambios en el uso del suelo y desertificación en el Mediterráneo: El caso del Sureste ibérico. En: GARCÍA RUIZ, J.M. y LÓPEZ GARCíA, P. (editores). Acción humana y desertificación en ambientes mediterráneos. Zaragoza: Instituto Pirenaico de Ecología, CSIOC, 1997, p. 9-39.

HÄNTZSCHEL, J.; GOLDBERG, V. \& BERNHOFER, C. GIS-based regionalisation of radiation, temperature and coupling measures in complex terrain for low mountain ranges. Meteorological Applications, 2005, Vol. 12, No 1, p. 33-42.

HARGREAVES, G. Simplified coefficients for estimating monthly solar radiation in North America and Europe. Technical report, departmental paper, Dept. of boil. and Irrig. Engrg. Of Utah. Logan: State University, 1994.

HARGREAVES, G.H. \& SAMANI, Z.A. Reference crop evapotranspiration from temperature. Applied Engineering in Agriculture, 1985, Vol. 1, N 2, p. 96-99.

HENGL, T.; HEUVELINK, G.B.M \& STEIN, A. A generic framework for spatial prediction of soil variables based on regression-kriging. Geoderma, 2004, Vol. 120, № 1-2, p. 75-93.

HIEMSTRA, P.H.; PEBESMA, E.J.; TWENHÖFEL, C.J.W. \& HEUVELINK, G.B.M. Realtime automatic interpolation of ambient gamma dose rates from the Dutch Radioactivity Monitoring Network. Computers \& Geosciences, 2008, Vol. 5, № 8, p. 1711-1721.

HOFIERKA, J. \& SURI, M. The solar radiation model for Open Source GIS: implementation and applications. In: Proceedings of the Open Source GIS-GRASS users conference, Trento, 2002.

INTERGOVERNMENTAL PANEL ON CLIMATE CHANGE (IPCC). Resumen para responsables de políticas en el Informe especial sobre la evaluación del grupo intergubernamental de expertos sobre el Cambio Climático. Cambridge: Cambridge University Press, 2013.

KIDRON, G.J. \& ZOHAR, M. Spatial evaporation patterns within a small drainage basin in the Negev Desert. Journal of Hydrology. 2010. Vol. 380, № 3-4, p. 376-385.

LLASAT, M.C. High magnitude storms and floods. In: WOODWARD, J.C. (editor). The Physical Geography of the Mediterranean. Oxford: Oxford University Press, 2009, p. 513-540.

LI, Z.; ZHENG, F.L. \& LIU, W.Z. Spatiotemporal characteristics of reference evapotranspiration during 1961-2009 and its projected changes during 2011-2099 on the Loess Plateau of China. Agricultural and Forest Meteorology, 2012, No 154-155, p. 147-155.

LÓPEZ BERMÚDEZ, F. y ALONSO SARRÍA, F. Aridez y sequías en la Cuenca del Segura. En: GIL OLCINA, A. y MORALES GIL, A. (editores). Causas y consecuencias de las sequías en España. Alicante: Universidad de Alicante y Caja de Ahorros del Mediterráneo, 2001, p. 187-205. 
LÓPEZ BERMÚDEZ, F.; CONESA GARCÍA, C. \& ALONSO SARRÍA, F. Floods: Magnitude and frequency in ephemeral channels of the Spanish mediterranean region. In: BULL, L.J. \& KIRKBY, M.J. (editors). Dryland rivers. Hidrology and Geomorphology of semi-arid channels. Chichester: John Wiley \& Sons Ltd., 2002, p. 329-350.

LÓPEZ BERMÚDEZ, F.; QUIÑONERO RUBIO, J.M.; GARCÍA MARÍN, R.; MARTÍN DE VALMASEDA GUIJARRO, E.; SÁNCHEZ FUSTER, M.C.; CHOCANO VAÑÓ, C. y GUERRERO GARCÍA, F. Fuentes y manantiales de la Cuenca del Segura. Murcia: Instituto Euromediterráneo del Agua y Confederación Hidrográfica del Segura, 2014.

LÓPEZ-URREA, R.; MARTín DE SANTA OLALLA, F.; FABEIRO, C. \& MORATALLA, A. Testing evapotranspiration equations using lysimeter observations in a semiarid climate. Agricultural Water $\mathrm{Ma}$ nagement, 2006, Vol. 85, Nº 1-2, p. 15-26.

MARDIKIS, M.; KALIVAS, D. \& KOLLIAS, V. Comparison of interpolation methods for the prediction of reference evapotranspiration. An application in Greece. Water Resources Management. 2005. No 19 p. 251-278.

MATHERON, G. The theory of regionalized variables and its applications. Paris: Les Cahiers du Centre de Morphologie Mathematique in Fontainebleu, 1971.

MILLÁN, M.; ESTRELA, J.M. \& CASELLES, V. Torrential precipitations on the Spanish east coast: The role of the Mediterranean sea surface temperature. Atmospheric Research, 1995, Vol. 36, № 1, p. $1-16$.

MINISTERIO DE MEDIO AMBIENTE (MMA). Principales conclusiones de la Evaluación Preliminar de los Impactos en España por efecto del Cambio Climático. Madrid: Centro de Publicaciones del Ministerio de Medio Ambiente, 2005.

MORALES GIL, A.; RICO AMORÓS, A.M. y HERNÁNDEZ HERNÁNDEZ, M. El trasvase Tajo-Segura. Observatorio Medioambiental, 2005, № 8, p. 73-110.

MORATA GASCA, A. Guía de escenarios regionalizados de cambio climático sobre España a partir de los resultados del IPCC-AR4. Madrid: Ministerio de Agricultura, Alimentación y Medio Ambiente, AEMET, 2014.

NAKICENOVIC, N.; ALCAMO, J.; DAVIS, G.; DE VRIES, B.; FENHANN, J.; GAFFIN, S. \& DADI, Z. IPCC special report on emissions scenarios. Cambridge: Cambridge University Press, 2000.

ODEH, I.O.A.; MCBRATNEY, A.B. \& CHITTLEBOROUGH, D.J. Spatial prediction of soil properties from landform attributes derived from a digital elevation model. Geoderma, 1994, Vol. 63, No 3-4, p. 197-214.

O'BRIEN, R.M.A. Caution regarding rules of thumb for Variance Inflation Factors. Quality \& Quantity, 2007, Vol. 41, N 45, p. 673-690. 
PETISCO DE LARA, S.E. Método de regionalización de temperaturas basado en análogos. Explicación y validación. Nota Técnica 3B del Área de Evaluación y Modelización del Cambio Climático (NT AEMCC-3B). Madrid: Agencia Estatal de Meteorología, 2008.

QUEREDA SALA, J.; MONTÓN CHIVA, E.; ESCRIG BARBERÁ, J.; RUESCAS, A.B. y MOLLÁ, B. La temperatura superficial del mar como señal de alerta en las grandes ciclogénesis mediterráneas. En: CHASTAGNARET, G. y GIL OLCINA, A. (editores). Riesgo de inundaciones en el mediterráneo occidental. Alicante: Casa de Velázquez - Universidad de Alicante, 2006, p. 91-114.

RODRÍGUEZ-PASCUA, M.A. y DE VICENTE, G. Análisis de la fracturación en materiales recientes (Mioceno superior-Cuaternario) en el Prebético Externo de Albacete. Boletín Geológico y Minero, 2001, Vol. 112, N 4, p. 65-77.

ROECKNER, E. ENSEMBLES ECHAM5-MPI-OM 20C3M run1, daily values. CERA database. Hamburgo: Word Data Center for Climate, 2007.

ROECKNER, E. ENSEMBLES STREAM2 ECHAM5C-MPIOM 20C3M run1, daily values. CERA database. Hamburgo: Word Data Center for Climate, 2008.

ROMERO DÍAZ, M.A. y LÓPEZ BERMÚDEZ, F. Erosión y desertificación en cuencas neógeno-cuaternarias de la Región de Murcia. Murcia: Instituto Euromediterráneo del Agua, 2009.

SAMANI, Z. Estimating solar radiation and evapotranspiration using minimum climatological data (Hargreaves- Sanami equation). Journal of Irrigation Drainage Engineering, 2000, Vol. 129, № 5, p. 360-370.

SHONGWE, M.; VAN OLDENBORGH, G.; VAN DEN HUR, B.; DE BOER, B.;COELHO, C. \& VAN AALS$\mathrm{T}, \mathrm{M}$. Projected changes in mean and extreme precipitation in Africa under global Warming. Part I: Southern Africa. Journal of Climate, 2009, Vol. 22, Nº 1, p. 3819-3837.

SHONGWE, M.; VAN OLDENBORGH, G.; VAN DEN HURK, B. \& VAN AALST, M. Projected changes in mean and extreme precipitation in Africa under global warming. Part II: East Africa. Journal of Climate, 2011, Vol. 24, N¹1, p. 3718-3733.

SUBBURAYAN, S.; MURUGAPPAN,A. \& MOHAN, S. Modified Hargreaves equation for estimation of $\mathrm{ET}_{0}$ in a hot and humid location in Tamilnadu State, India. International Journal of Engineering Science and Technology, 2011, Vol. 3, No 1, p. 592-600.

TAO, X.E.;CHEN, H.; XU, C.Y.; HOU, Y.K. \& JIE, M.X. Analysis and prediction of reference evapotranspiration with climate change in Xiangjiang River Basin, China. Water Science and Engineering, 2015, Vol. 8, N 4, p.273-281.

TERINK, W.; IMMERZEEL, W.W. \& DROOGERS, P. Climate change projections of precipitation and reference evapotranspiration for the Middle East and Northern Africa until 2050. International Journal of Climatology, 2013, Vol. 33, N 14, p. 3055-3072. 
VAN DER LINDEN, P. \& MITCHELL, J.F.B. ENSEMBLES: Climate Change and its Impacts: Summary of research and results from the ENSEMBLES project. Exeter: Met. Office Hadley Centre, 2009.

VICENTE-SERRANO, S.M.; AZORÍN-MOLINA, C., SÁNCHEZ-LORENZO, A.; REVUELTO, J.; LÓPEZ-MORENO, J.I.; GONZÁLEZ-HIDALGO, J. C. \& ESPEJO, F. Reference evapotranspiration variability and trends in Spain, 1961-2011. Global and Planetary Change, 2014, No 121, p. 26-40.

VICENTE-SERRANO, S.; LANJERIB, S. \& LÓPEZ-MORENO, J.I. Comparison of different procedures to map reference evapotranspiration using geographical information systems and regression-based techniques. International Journal of Climatology, 2007, Vol. 27, № 8, p.1103-1118.

VICENTE-SERRANO, S; AZORÍN-MOLINA, C.; SÁNCHEZ-LORENZO, A; EL KENAWY, A.; MARTÍN-HERNÁNDEZ, A.; PEÑA-GALLARDO, M.; BEGUERÍA, S. \& BURGUERA, M. Recent changes and drivers of the atmospheric evaporative demand in the Canary Islands. Hydrology Earth System Sciences, 2016, N²0, p. 3393-3410. 